\title{
Attention Attraction in Circular Perception of a Three- Dimensional Object
}

\author{
Losik George ${ }^{1 *}$, Boyko Igor ${ }^{1}$, Tkachenko Vadim ${ }^{1}$, Stralchonak Oleg ${ }^{1}$, Vilchuk Uri ${ }^{2}$ and Gladkaya Victory ${ }^{2}$ \\ ${ }^{1}$ United Institute for Informatics Problems of the NAS of Belarus, Belarus
}

${ }^{2}$ Belarusian State University of Informatics and Radioelectronics, Belarus

*Corresponding author: Losik George, United Institute for Informatics Problems of the NAS of Belarus, Belarus.

\section{Abstract}

The article is dedicated to the parametric analysis of a trajectory of circular rotation of a three-dimensional object during its perceptual inspection and emphasizes the difference in the psychological mechanism of distribution of a person attention from the mechanism of saccadic eye movement during perception of the video scene. According to the result, when the image of the three-dimensional object scanned with eyes there are saccades and fixed points of attention. The difference between perception of a physical object and its virtual image lays in necessity of physical action to go around the object or rotate it with the hand. So, there is a hypothesis the motive to form a full virtual image of the object consists of consequence of discrete motives, like: look at the object from left, from right, from behind, from top. In that case a person has a freedom of choice which discrete motive to implement and do it consciously. Perception of parameters of the object occurs during its rotation with an accent on the points of interest. In that points attention attracted and kept for some time, comprehension of perceived scene occurred, and the new motive generated. In the point of interest, the observation trajectory makes a maneuver and this fact support the hypothesis. The maneuvers reflect change of cognitive motives. The topology of the object image formed as a graph with vertices and vectors with azimuths and lengths of vertex connections as vector-defined edges of the graph. The results of the study are applicable in ergonomic design and prediction of human perceptual actions in new cognitive situations.

Keywords: 3D object; Attention attraction; Cognitive motive; Perception trajectory; Point of interest

\section{Introduction}

Now days there are task of the information search of 3D images on the Internet [8;12]. Similar to the textual search by a key word there is a goal to find invariant properties of topology of the 3D object shape to search not just copies of the object but objects similar by the shape. In that case it is not enough to consider only physical similarity of the objects but psychological perception should be taken into the count. So, psychologists proposed to use eye-tracking. We propose to use maps of trajectories of circular perception of the object. Comparing such trajectories and considering speed of observation the Internet search system could find object by similar shape and psychological style of observation simultaneously. It gives a benefit to place, for example, virtual 3D sculptures in a museum or 3D goods on the Internet market with consideration of a psychological component of virtual information search.

There is a large number of researches in the trajectory perception of planar or 2D images. Regularities of viewing 2D objects were studied by [1-5]. The Eye-tracking method allows studying the patterns of eye movements and stops when viewing 2D images [6]. Real or virtual objects on the computer display may be taken for viewing. Valuable for analysis are the "heat maps" as statistically averaged data on the planar spectrum of visual attention to different spots on the image (Figure 1).

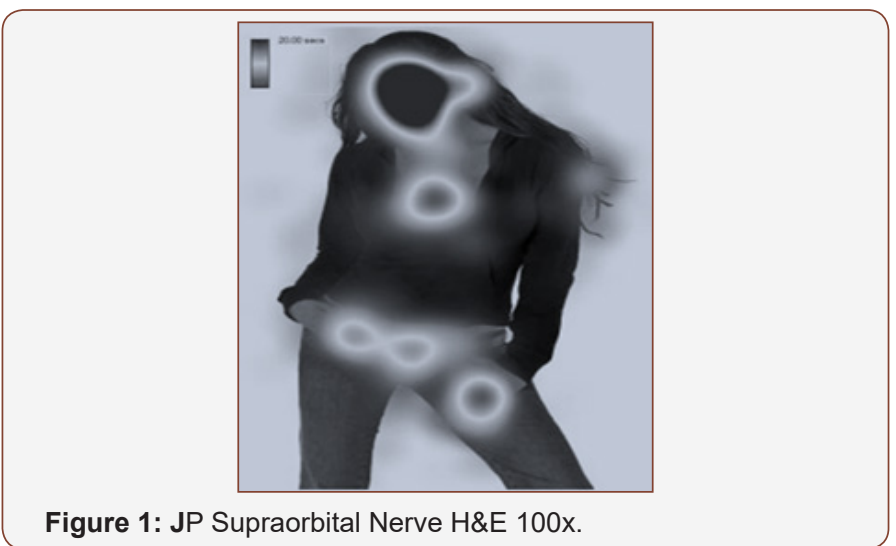


However, these researches on 2D objects do not include perception of the rotation trajectories of 3D non-transparent objects. The 3D object perception demands viewing the object around and it is forming the trajectory in the 3D space unlike to 2D space (Figure 2). To inspect the entire surface of the real object, the person has to walk around the object or rotate it with the hand [7].The perception of a route as the trajectory in the 3D space considered being analog and changing in time and speed. During the human perception of the 3D object surface, the viewing trajectory does not contain irregular gaze leaps (Figure 2).

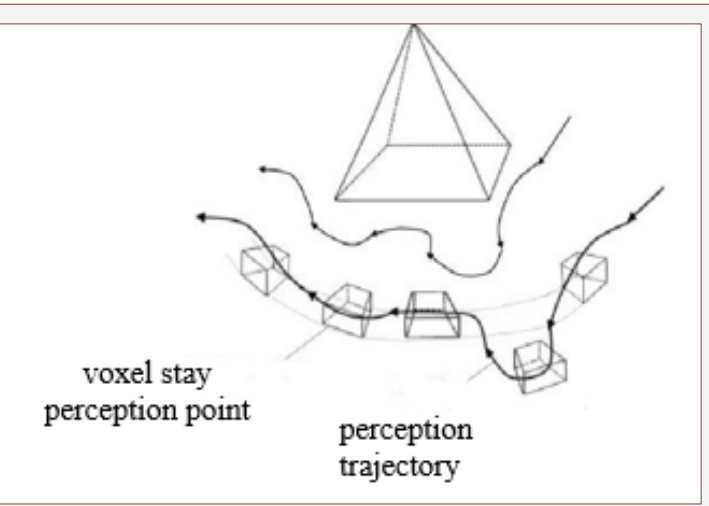

Figure 2: The viewing trajectory

So, a person's hand or body is involved to rotate the object, then the trajectory of the perception depends not only on a direction of the person attention but also on the mobility of the hand and body. There are three reasons affecting the circular trajectory:

- the physical properties of the inspected object

- the physiological characteristics of the person (hand, body)

- $\quad$ the psychological motives

Particular motives can determine the nature of the perception. These are different approaches when the object is inspected purely for curiosity (cognitively) and when the person inspects the object for pragmatic purposes where a phase of perception is preceding the phase of the immediate specific use of this object. This paper considers only the first approach [8].

In the experiment, the person did not have an access to the real object, instead had the access to the 3D model on the screen. The person rotated the object with the hand and the "computer mouse". So, the person could not plan any instrumental actions with the object, because did not have sensations of the object's weight, center of gravity, aggregate state, roughness, temperature, elasticity perceiving the object on the basis of cognitive motives only.

The results of the previous research $[9,10]$ show that observation of the 3D object during its rotation has a similarity with observation of the 2D object because of stops of attention of saccades. The novelty of the research is in recognition of psychological factors affecting the perception trajectory which are dipper of physiological and physical factors. Besides, the novelty is in developed algorithms and software registering the perception trajectory and recognizing cognitive motives of the person so supporting the statement of informatively of the factors.

\section{Materials and Methods}

The origin of the motive is related to two sources. The first when an intention of the person is based on the received instruction and formed before the object perception. The second when in natural conditions there is some source "inside" of the person and based on that there is the motive. This research is concentrated on the second case [11]. The experiments showed that the motive has many variants and such a variability arises on four reasons dependent on:

- the characteristics of the inspected object (its physical properties)

- the physiological patterns of sensorimotor control over the movements of the hand and eye

- the content of the instruction to the person

- the psychological intention of the person fulfilled during the perception

The viewing trajectory is the result of the interaction of those four factors. The first three factors shadow the fourth. The fourth factor, which is most interesting, can be, for example, the person's motive to see the backside of the object ("to look beyond the horizon"); or to approach to the point of the focal interest; or to examine the frontal panorama of the object; or to understand whether the object is symmetrical. Therefore, before automatic motive recognition it is necessary to "eliminate" from the "raw" trajectory factors reflecting the physics of the object and the physiology of the person's hand and eye. According to the identified reasons for the implementation of the recognition method, it was developed an algorithm of forming and filtering the average trajectory from the registered trajectories of the virtual object perception. It is on the filtered trajectory the sections corresponding to the implementation of the motive [12].

For that, in the psychology the classic approach is used to the factor analysis, variance analysis, and structural modeling methods [11]. In the presented research, a method of counter hypotheses is applied. In this method, decomposition of the causes of variability is accomplished not by a mathematical method, but by the method of controlling the experiment - by eliminating the hypothesized factor in the experiment.

The person can have different psychological intentions during the object perception. In the research, only one cognitive hypothesis is tested: "to approach to the focal interest point." On the surface of the object presented to the person - "the globe" - the five yellow spots were placed as the points of interest. According to the instruction, the person was required to find those points in a random order and to make short-term stops during the perception [9].

\section{The test plan}

For the experiments was provided a laboratory model, developed in the United Institute of Problems of Informatics of 
Belarus Academy of Science, registering the perception trajectory of the 3D object and helping to define a set of parameters. To study the patterns of the 3D object inspection, in a pilot experiment from 50 samples of 3D models only one was selected. This is the globe. The continents and the water areas are depicted as a "physical map" without conventional notations. On the globe surface, 5 yellow spots were marked for searching. Before the experiment, for adaptation, the participant was asked to rotate another object with the mouse and after that, the instruction was given. The position to begin the inspection was the same for everybody - the western hemisphere. Each person made experience once. There were no restrictions on the search duration and sequence of the yellow spots $[13,14]$. Thus, variety of inspection conditions were allowed with respect to the azimuth, route of inspection, and speed (accelerations, stops, and time dynamics of the inspection). With the mouse wheel the participant could magnify the point of interest on the surface. 150 participants 16-21 years old performed the test. 70\% are students of BSPU and $30 \%$ are high school students. All having normal vision and right-handers.

\section{Results}

The globe surface is divided on 512 triangles. A red line going through some of the 512 triangles and representing the attraction spectrum marks the inspection trajectory of the participants. (Figure 3) The time of being in the triangles is called the spectrum time of attention attraction to the different spots of the object (Figure 3).

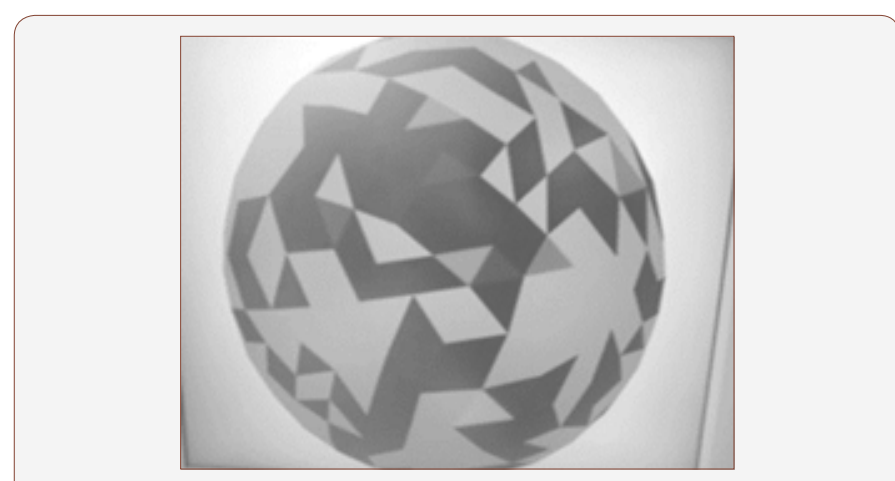

Figure 3: The spectrum of attention attraction to triangles distributed on the surface of the globe.

The spectrum reflects only attention attraction to some spots but not provide statistics of the direction of the participant viewing movement. So, the statistics of vectors, showing the direction from the particular triangle to the particular, was collected. The result is called the spectrum of viewing directions. As noted, the globe surface is divided on 512 triangles. The result of averaging data of 150 participants on the time of stops in each of the 512 triangles is the attention attraction spectrum represented as a histogram (Figure 4).

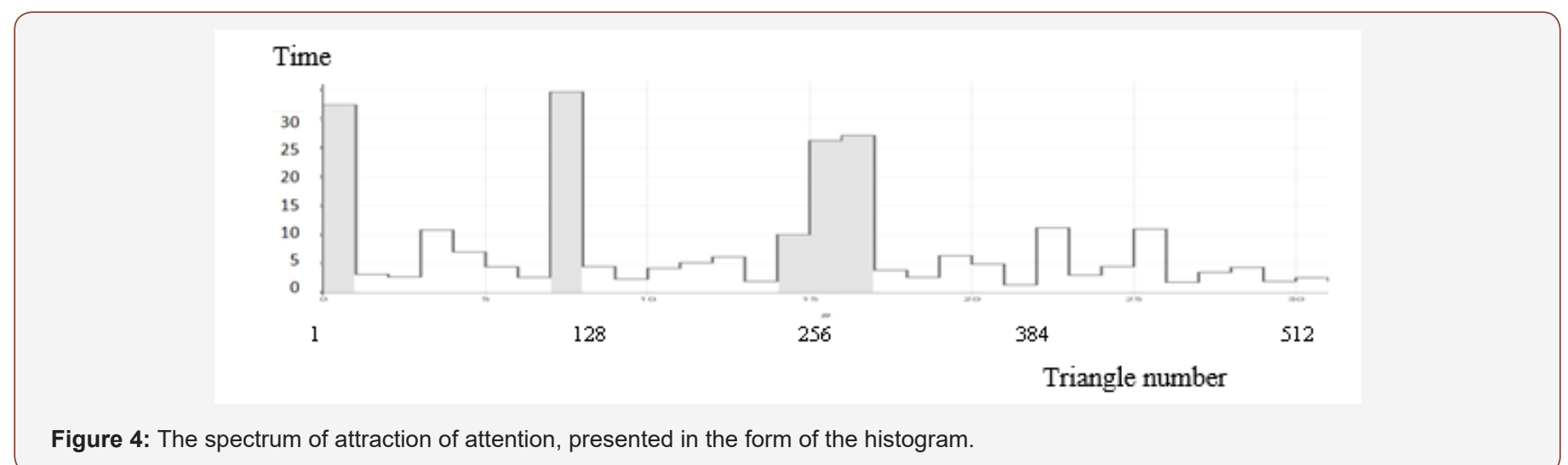

It was detected the time of stops in different places on the sphere is varying much. There are places attracting attention and not attracting. In particular, all the five yellow spots pointed to be places of attention, included in such places. Besides, there is a number of unplanned detected places on the surface of the globe. It is a matter of next experiments to learn how automatically predict which places will attract more attention on other type of objects. The spectrum shows only attraction of the gaze to a particular place on the surface of the object. So, the participants made stops in places where they found yellow spots and stops in a number of other places interesting for the participants. It was assumed that the type of the object affects not only the duration of perception of different places on the object surface, but also affects the vector (direction) of the gaze leaving the place to the favor of the neighboring place, and the speed of exit. So, the places of interest, were defined according to a condition of the diversity or monotony of the directions of entry and exit from the given place. In a result, the statistical averaging of the perception trajectories of the same object by a number of participants allowed to classify different places into three categories: hubs (the places of high interest); paths between hubs; not visited places.

According to the instruction, the five yellow spots were identified as places of interest. Each spot lays on the several triangles. Therefore, the vectors and frequencies of entry and exit were statistically computed and the frequency of the entry directions in the spots and exit directions from the spots were compiled. So, the important parameter is the direction of entry to the spot and exit from the spot. The attention attraction time is not enough to characterize the triangle as a hub. The hub is characterized by the factor of a set of different directions of entry and exit but not just a single one direction. All five yellow spots satisfy to the factor. The average parameter was used to select the 
frequency of movement of the gaze from the ith triangle to the neighboring jth, which is a specific pair of neighboring triangles. Considering that the inspection sphere consists of 512 triangles, $512 \times 512$ pairs of triangles were allocated for statistical analysis and $512 \times 512$ matrix was compiled. Statistics were collected for

Table 1: The number of triangles being hubs for 150 participants.

\begin{tabular}{|c|c|c|c|}
\hline The number of the yellow spot & $\begin{array}{c}\text { The number of the triangle for } \\
\text { the spot }\end{array}$ & $\begin{array}{c}\text { Total number of exits from the } \\
\text { spot in all directions }\end{array}$ & $\begin{array}{c}\text { The number of different exit } \\
\text { directions }\end{array}$ \\
\hline 1 & 2 & 3 & 4 \\
\hline 1 & 9 & 150 & 9 \\
\hline 2 & 127 & 150 & 13 \\
\hline 3 & 220 & 150 & 10 \\
\hline 4 & 249 & 150 & 14 \\
\hline 5 & 268 & 150 & 16 \\
\hline Additional 1 & 60 & 93 & 17 \\
\hline Additional 2 & 380 & 88 & 19 \\
\hline Additional 3 & 410 & 98 & 21 \\
\hline
\end{tabular}

According to the instruction, the five yellow spots were identified as places of interest. Each spot lays on the several triangles. Therefore, the vectors and frequencies of entry and
150 experiments. For each participant the detected trajectory was transcoded into a chain of a sequence of triangles and pairs of adjacent triangles were found. For each triangle, which could potentially be a place of change of motive or hub, the number of exit triangles was determined (Table 1). exit were statistically computed and the frequency of the entry directions in the spots and exit directions from the spots were compiled (Figure 5).

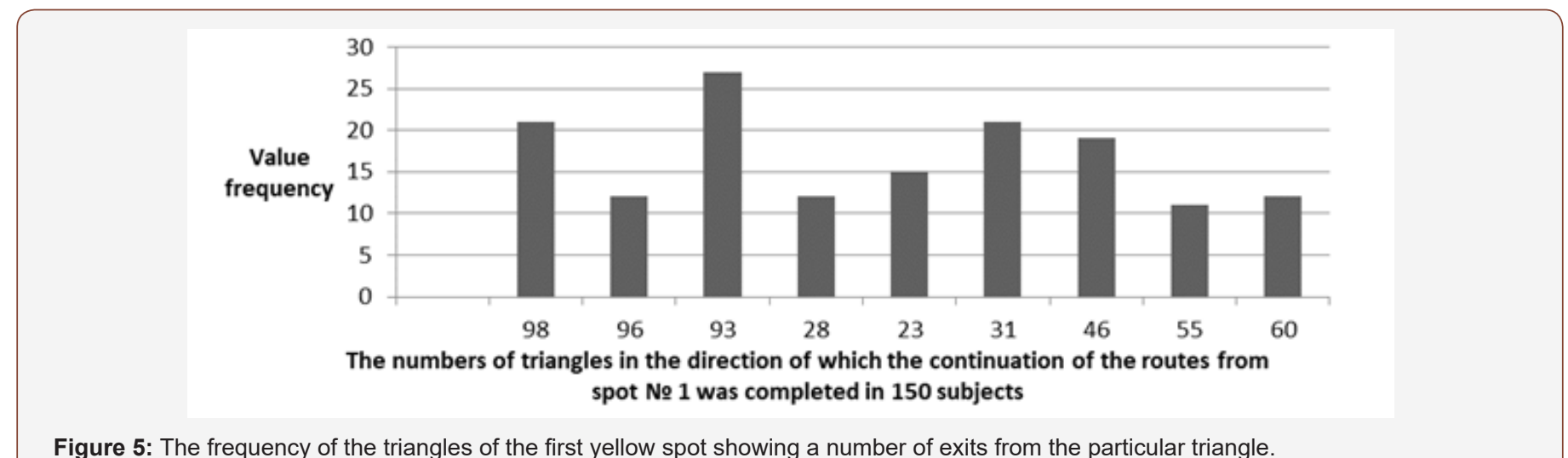

Figure 5: The frequency of the triangles of the first yellow spot showing a number of exits from the particular triangle.

So, the important parameter is the direction of entry to the spot and exit from the spot. The attention attraction time is not enough to characterize the triangle as a hub. The hub is characterized by the factor of a set of different directions of entry and exit but not just a single one direction. All five yellow spots satisfy to the factor. The number of exits from the first spot is 17 , from the second is 14 , from the third is 9 , from the forth is 25 . That means the hub is place of completion of the one motive and beginning of another.

\section{Discussion}

The study revealed two reasons affecting the change of route of the circular perception of the object. The first is a specificity of the object and the second is the specificity of the focus group of the participants. Therefore, when changing the object of perception, it should be expected a difference of the places of interest on the surface of the object, a different duration of stops, a different angular speed of rotation of the object from the point to point. Change of the category of participants leads to differentiation of sequences of points of interest and directions of attention.
Thus, accumulated information about the time of the person attention to certain places on the surface of the object and information about the variety of exit directions from these places, can help automatize finding places on the route that are interesting for the perception on the surface of the object. Theoretical analysis showed that the circular perception of the nontransparent object is fundamentally different in nature from the perception of a plane object by the saccades of the eyeball. The perception is associated with stops of attention to comprehend the point of interest, a time delay for choosing a further inspection of the object by rotation.

The trajectory of the circular perception of the object shows the place of interest where the person makes a maneuver and attention is kept. Maneuvers along the route when the object rotates in a new direction can act as informative signs of places of changing the cognitive motive of a person in a circular perception of the object. This does not contradict the hypothesis that the consideration in the image the parameters of the 3D object on its rotation path occurs only in interesting places of the object and the new inspection motive begins to be realized after the comprehension of 
the interesting point. At the same time, the participant remembers the appearance of the object in this interesting place to not return there again.

\section{Conclusion}

So, achieved results do not contradict the hypothesis that during circular perception of the 3D object the person finding the point of interest decides about the next maneuver, what means changes the motive of observation. At the same time, the person memorizes the view of the object in the point of interest to avoid coming back to the point again.

\section{Acknowledgement}

None

\section{Conflicts of Interest}

No conflict of interest.

\section{References}

1. Tversky B (2017) Thinking inside and outside. In L Magnani and T Bertolotti (eds), Handbook of model-based science, New York, p. 345.

2. Zinchenko VP (1971) Productive perception. Questions of Psychology 6: 19-29.

3. Gibson J (1955) Perceptual learning-differentiation or enrichment? Psychol Rev 62(1): 32-41.
4. Antipov VN, Jegallo AV (2014) 3D perception of planar images in computerized environment. Experimental psychology 3: 97-111.

5. Lahlou S (2011) How can we capture the subject's perspective? Social Science Inform 50: 607-655.

6. Chen L (2005) The topological approach to perceptual organization. Psychology Press 12(4): 553-637.

7. Goncharov OA (2007) Perception of space and perspective constructions. SPb: SPbSU p. 213 c.

8. Caraux G, Pinloche S (2005) Permut Matrix: a graphical environment to arrange gene expression profiles in optimal linear order. Bioinformatics 21(7): 1280-1281.

9. Losik GV (2015) Coding Information in the Brain Monograph. Lap Lambert Academic Publishing, Europe, p. 135.

10. Losik GV, Derugin AA, Boyko IM, Tkachenko VV, Sivak SA (2017) The strategy of observation of a 3D virtual object surface. RINTI UIPI NASB, Minsk, Belarus, pp. 392-397.

11. Losik GV (2008) Perceptual actions of a person: cybernetic aspect. Minsk: OIPI NAS of Belarus, p. 147.

12. Lebedev AN (2001) The oscillatory mechanism of memy. Intern Quarterly of Cogn Sciences 2: 57-66.

13. Losik GV (2014) Analog coding a shape of an object in the human brain. RINTI-UIPI NASB, Minsk, Belarus, pp. 157-162.

14. Losik GV, Severin A, Asadchy Y (2016) The Perception of Object with Flexible Shape by Visually Impaired Persons. Sensory issues and Disability: Proc of intern Conf, Paris, France, p. 37. 